\title{
Optimal Sequencing of Inspection of Dependent Characteristics ${ }^{1}$
}

\author{
S. O. Duffuua ${ }^{2}$ And S. M. Pollock ${ }^{3}$ \\ Communicated by G. P. Papavassilopoulous
}

\begin{abstract}
We address the problem of sequentially inspecting the dependent characteristics of a product, where the dependency is expressed in terms of the joint probabilities of the fitness of the characteristics. We show that, even when the inspection has classification errors, the joint probability mass function of the observed fitness of the characteristics is independent of the sequence of inspection. Using this result, a dynamic programming approach is presented for finding the optimal sequence that minimizes the expected total cost of inspection. Previously reported policies for independent characteristics are shown to be special cases of the results presented here.
\end{abstract}

Key Words. Multicharacteristic inspection, sequencing, dynamic programming.

\section{Introduction}

In the multicharacteristic inspection problem, products (referred to in the sequel as "components") with several characteristics must be inspected (Refs. 1,2). These characteristics have different effects on the component fitness for use; indeed, some are of critical importance to human life (Ref. 2). For any produced component, inspection of each characteristic must be

'The first author acknowledges the support provided by King Fahd University of Petroleum and Minerals during his 1994-95 sabbatical leave and by the Department of Industrial Engineering of the University of Michigan for extending its facilities to him at that time. The second author acknowledges partial support from the University of Michigan Flat Plate Display Technology Center.

${ }^{2}$ Professor, Systems Engineering Department, King Fahd University of Petroleum and Minerals, Dhahran, Saudi Arabia.

${ }^{3}$ Professor, Department of Industrial and Operations Engineering, University of Michigan, Ann Arbor, Michigan. 
carried out. However, inspection is costly, depending on the type of test anc equipment needed to carry out the tests. Moreover, these tests are imperfect and have associated with them the usual type I and type II errors.

An important and realistic situation is one where the inspection of a component characteristics can be carried out sequentially. The component is rejected if one characteristic is observed to be defective; if all characteristics pass inspection, the component is accepted. The problem of ordering the characteristics for such sequential inspection was addressed in Ref. 2, using the assumption that the fitness of the characteristics were statistically independent; this led to the independent multicharacteristic sequencing problem (IMIP). Under this assumption, the policy that minimizes the expected inspection cost per accepted component was shown to be (Ref. 2) "inspect the characteristics in increasing order of the ratio of the cost of inspection to the probability of rejection," where the probability of rejection is a simple function of the probabilities of fitness and inspection errors. This policy is similar in structure to those commonly found in machine scheduling problems (Ref. 3). In Ref. 4, the IMIP is stated in more general terms, a necessary and sufficient condition is derived for the optimality of this sequencing rule, and many examples are cited as special cases of this more general model. The stochastic sequencing problem (Refs. 2, 4), a further generalization of the IMIP, allows some input variables to be random. The associated optimal sequencing rule is obtained in Ref. 5 .

This paper generalizes the IMIP by relaxing the assumption tnat the fitness of characteristics are statistically independent; i.e., we analyze the dependent sequencing problem (DSP). Many situations exhibit such dependence. For example, an applicant joining the police or the armed forces must be examined medically for blood pressure, weight, and heart function; yet, these are clearly statistically dependent characteristics. Another example involves the final inspection of an air conditioner, in which there are 14 inspectable characteristics (e.g., inner temperature, outer temperature, thermostat response, compressor current draw, etc.) most of which are statistically dependent.

In particular, we develop a dynamic programming (DP) approach for finding the optimal sequencing policy (i.e., one that minimizes the expected cost of inspection) for the DSP. The motivation for using DP is that it provides an immediate structural form for the optimal inspection policy, one which reduces to the rule given in Ref. 2 for independent characteristics.

The rest of the paper is organized as follows. In Section 2, the problem is defined precisely. Section 3 presents the joint probability mass function for the observed fitness and its properties. Section 4 provides the DP formulation. Section 5 contains the conclusions. 


\section{Statement of the Problem}

We are given a component with $N$ characteristics, each of which is either defective (D) or fit (F). For each characteristic, it is assumed that we know

$$
P_{i}=\operatorname{Pr}\{\text { characteristic } i \text { is defective }\} .
$$

For convenience, we use the indicator random variable $W_{1}$, such that

$$
\begin{aligned}
& W_{1}=0, \quad \text { when characteristic } i \text { is } D, \\
& W_{1}=1, \quad \text { when characteristic } i \text { is } F .
\end{aligned}
$$

Since we are concerned about the case where the characteristics are dependent, this is equivalent to recognizing that the random variables $W_{1}$ are dependent. In particular, we assume that we know $P_{W}\left(w_{1}, w_{2}, \ldots, w_{N}\right)$, the joint probability mass function of the components of the fitness vector $W \equiv\left(W_{1}, W_{2}, \ldots, W_{N}\right)$. The marginal probability mass function for $W_{1}$ is

$$
P_{W_{1}}\left(w_{1}\right)=\sum_{w,=0,1, j \neq i} P_{W}\left(w_{1}, w_{2}, \ldots, w_{N}\right),
$$

and so, by definition,

$$
P_{1}=P_{w_{1}}(0) \text {. }
$$

Each characteristic $i$ can be inspected at a cost $c_{i}>0$. The inspection of characteristic $i$ produces an observation $X_{i} \in\{0,1\}$ such that:

(a) if $X_{i}=0$, the component is declared defective with respect to that characteristic; e.g., it is rejected and not subject to further inspection;

(b) if $X_{1}=1$, the component is declared fit with respect to that characteristic, and another characteristic is chosen to be inspected.

If all characteristics are declared fit, the component is accepted.

Due to the stochastic nature of the test, $X_{i}$ is a random variable, which leads to type I and type II errors defined as

$$
\begin{aligned}
\alpha_{1} & \equiv \operatorname{Prob}\left\{X_{i}=0 \mid W_{i}=1\right\} \\
& =\operatorname{Prob}\{\text { classifying characteristic } i \text { as defective when it is fit }\}, \\
\beta_{i} & \equiv \operatorname{Prob}\left\{X_{i}=1 \mid W_{i}=0\right\} \\
& =\operatorname{Prob}\{\text { classifying characteristic } i \text { as fit when it is defective }\} .
\end{aligned}
$$


In the above, we assume implicitly that the distribution of $X_{i}$ is independent of $W_{j}$, for $j \neq i$. However, the $X_{i}$ themselves are not necessarily mutually independent, and for that reason we introduce the following notation:

$$
\begin{aligned}
& q_{X}\left(x_{1}, x_{2}, \ldots, x_{N}\right) \\
& \equiv \text { joint probability mass function of } X \equiv\left(X_{1}, X_{2}, \ldots, X_{N}\right) .
\end{aligned}
$$

The fundamental problem is to find the optimal sequence of inspections, that is, the sequence that minimizes the expected cost of inspection per component. For small values of $N$, it is feasible to compute the expected cost of inspection for all $N$ ! sequences, and from that to find the optimal sequence. However, as $N$ becomes large, this total enumeration approach becomes infeasible. It is not uncommon in industry to have a component with $N=15$ characteristics which results in over $10^{12}$ sequences to be considered.

\section{Joint Probability Mass Function of the Observations}

In this section, an expression for $q_{X}\left(x_{1}, x_{2}, \ldots, x_{N}\right)$ is obtained for $x_{i} \in\{0,1\}, i=1,2, \ldots, N$. It has been shown earlier (Ref. 3 ) that, when the $W_{i}$ are independent, this function is independent of the order of the sequence of inspection. We show that this is true even for the dependent case. We also show that computing $q_{X}\left(x_{1}, x_{2}, \ldots, x_{N}\right)$ involves complexity of at most $N 2^{N}$.

For convenience, we first define the function $\delta_{i}(x)$ such that

$$
\begin{aligned}
& \delta_{i}(0)=\alpha_{i}, \\
& \delta_{i}(1)=\beta_{i} .
\end{aligned}
$$

When $N=1$, the probability mass function for the observation $X_{1}$ is

$$
q_{X_{1}}\left(x_{1}\right)=\delta_{1}\left(x_{1}\right) P_{W_{1}}\left(1-x_{1}\right)+\left[1-\delta_{1}\left(1-x_{1}\right)\right] P_{W_{1}}\left(x_{1}\right) .
$$

When $N=2$, conditioning on all possible values of $w_{1}$ and $w_{2}$ produces

$$
\begin{aligned}
q_{X_{1}, X_{2}}\left(x_{1}, x_{2}\right)= & {\left[1-\delta_{1}\left(x_{1}\right)\right]\left[1-\delta_{2}\left(x_{2}\right)\right] P_{W_{1}, W_{2}}\left(x_{1}, x_{2}\right) } \\
& +\delta_{1}\left(1-x_{1}\right)\left[1-\delta_{2}\left(x_{2}\right)\right] P_{W_{1}, W_{2}}\left(1-x_{1}, x_{2}\right) \\
& +\left[1-\delta_{1}\left(x_{1}\right)\right] \delta_{2}\left(1-x_{2}\right) P_{W_{1} W_{2}}\left(x_{1}, 1-x_{2}\right) \\
& +\delta_{1}\left(1-x_{1}\right) \delta_{2}\left(1-x_{2}\right) P_{W_{1}, W_{2}}\left(1-x_{1}, 1-x_{2}\right) .
\end{aligned}
$$


Note that this formula incorporates all possible combinations of $x_{i}$ and $1-x_{i}, i=1,2$, in the argument of $P_{W_{1}, W_{2}}(\cdot, \cdot)$. More importantly, the conditioning does not depend on the order of inspections (i.e., whether or not $X_{1}=x_{1}$ is observed before $X_{2}=x_{2}$, or vice versa).

The formula for $q_{X_{1}, X_{2}}\left(x_{1}, x_{2}\right)$ can be generalized easily for $X=$ $\left(X_{1}, X_{2}, X_{N}\right)$ by first defining the set

$$
\begin{array}{r}
S_{i} \equiv\left\{i: x_{i} \text { in the argument of } q_{X}(\cdots)\right. \text { equals } \\
\left.w_{i} \text { in the argument of } P_{W}(\cdots)\right\} .
\end{array}
$$

Then, a straightforward extension of the analysis leading to Eq. (2) yields

$$
\begin{aligned}
q_{X}\left(x_{1}, x_{2}, \ldots, x_{N}\right)= & \sum_{S_{i}} P_{W}\left(z_{1}, z_{2}, \ldots, z_{N}\right) \\
& \times \prod_{m \in S_{1}}\left[1-\delta_{m}\left(x_{m}\right)\right] \prod_{j \notin S_{1}} \delta_{j}\left(1-x_{j}\right),
\end{aligned}
$$

where the variables $z_{k}$ are defined such that

$$
\begin{aligned}
& z_{k}=x_{k}, \quad \text { if } k \in S_{i}, \\
& z_{k}=1-x_{k}, \quad \text { if } k \notin S_{i} .
\end{aligned}
$$

Proposition 3.1. The joint probability mass function $q_{X}\left(x_{1}, x_{2}\right.$, $\left.\ldots, x_{N}\right)$ of the observed characteristic fitnesses does not depend on the order of inspection.

This is evident by inspecting the right-hand side of Eq. (3) above.

Proposition 3.2. The computational complexity of producing $q_{X}\left(x_{1}\right.$, $\left.x_{2}, \ldots, x_{N}\right)$ is no greater than $N 2^{N}$.

This follows by noting that, for each of the $2^{N}$ realizations of $\left(x_{1}, x_{2}, \ldots, x_{N}\right)$, the right-hand side of Eq. (3) involves the sum of at most $N$ terms.

\section{Dynamic Programming Approach to the Dependent Sequencing Problem}

An analogy exists between the classical traveling-salesman problem and our multi-characteristic sequencing problem. If we represent the $N$ characteristics by $N$ cities to be visited, and the inspector by the salesman, the problem becomes one of sequentially deciding which city to visit next. One difference is that the inspector will terminate the tour as soon as a defective characteristic is encountered (i.e., an observation of $X_{i}=0$ is made). Otherwise, the 
inspector will proceed to another city (characteristic) if $X_{i}=1$. Another obvious difference is that the inspector does not return back to the first characteristic.

The analogy suggests that the sequencing problem lends itself to a DP formulation similar to one that can be constructed for the traveling-salesman problem (Ref. 6). Such a formulation is not useful for the direct computation of the minimum inspection cost when $N$ is large. However, it does provide a structural result that specifies the form of the optimal sequential inspection sequence. To show this, let:

$C=\{1,2, \ldots, N\}=$ set of all characteristics;

$\Theta=$ any subset of $C$ with cardinality $i$;

$f_{i}(\Theta) \equiv$ optimal value function $=$ minimum expected inspection cost per accepted component with $i$ yet-to-be-inspected characteristics in $\Theta$, given that all the characteristics in $\bar{\Theta} \equiv\{C \backslash \Theta\}$ have been inspected and classified as fit.

For convenience, we define the probability that an inspected characteristic will be classified as fit, given that all inspected characteristics in $\bar{\Theta}$ have been classified as fit, to be

$$
p_{k}(\Theta) \equiv \operatorname{Prob}\left\{X_{k}=1 \mid x_{i}=1, \forall i \in \bar{\Theta}\right\} .
$$

Using this definition, we can write the dynamic program

$$
f_{i}(\Theta)=\min _{k \in \Theta}\left[p_{k}(\Theta) f_{i-1}(\Theta \backslash\{k\})+c_{k}\right], \quad i=1,2, \ldots, N-2,
$$

with boundary condition (where $\Phi \equiv$ the empty set)

$$
f_{0}(\Phi)=0 \text {. }
$$

The solution $f_{N}(C)$ provides the optimal sequence in which characteristics should be inspected.

Note that, when $\Theta \neq C$, the values of $p_{k}(\Theta)$ can be computed from Eq. (3) by using the Bayes theorem, i.e.,

$$
p_{k}(\Theta)=a_{k}(\Theta) / b(\Theta)
$$

where

$$
\begin{aligned}
a_{k}(\Theta) \equiv & \operatorname{Prob}\left\{X_{k}=1 \cap X_{i}=1, \forall i \in \bar{\Theta}\right\} \\
= & \sum_{t_{j} \in\{0,1\}, \forall j \in \Theta \backslash\{k\}} q_{X}\left(t_{1}, t_{2}, \ldots, t_{N}\right), \\
& \quad \text { where } t_{m}=1, \forall m \in[\bar{\Theta} \cap\{k\}],
\end{aligned}
$$




$$
\begin{aligned}
b(\Theta) & =\operatorname{Prob}\left\{X_{i}=1, \forall i \in \bar{\Theta}\right\} \\
& =\sum_{t, \in\{0,1\}, \forall j \in \Theta} \mathrm{q}_{X}\left(\mathrm{t}_{1}, \mathrm{t}_{2}, \ldots, \mathrm{t}_{N}\right), \text { where } t_{m}=1, \forall m \in \bar{\Theta} .
\end{aligned}
$$

With no previous inspections, $\Theta=C$, and thus $\bar{\Theta}=\Phi$, in which case

$$
\begin{aligned}
& a_{k}(C)=\operatorname{Prob}\left\{X_{k}=1\right\}=P_{k}\left(1-\beta_{k}\right)+\left(1-P_{k}\right) \alpha_{k}, \\
& b(C)=1 .
\end{aligned}
$$

This formulation provides the following structural result for the optimal inspection policy.

Theorem 4.1. Suppose that, in some sequence of inspections,

(a) a set $\bar{\Theta} \subseteq C$ has been inspected;

(b) $k \in \Theta$ and $j \in \Theta$ are any two candidate characteristics for the next inspection;

(c) inspecting characteristic $k$ before characteristic $j$ will be preferred to (or no worse than) inspecting characteristic $j$ before characteristic $k$.

Then,

$$
c_{k} /\left[1-p_{k}(\Theta)\right] \leq c_{j} /\left[1-p_{j}(\Theta)\right] .
$$

\section{Proof.}

(i) Under condition (c), the right-hand side of Eq. (6) requires that

$$
p_{k}(\Theta) f_{i-1}(\Theta \backslash\{k\})+c_{k} \leq p_{j}(\Theta) f_{i-1}(\Theta \backslash\{j\})+c_{j} .
$$

(ii) If characteristic $j$ is inspected after characteristic $k$, the resulting value function is

$$
f_{i-1}(\Theta \backslash\{k\})=p_{j}(\Theta \backslash\{k\}) f_{i-2}(\Theta \backslash\{k\} \backslash\{j\})+c_{j} .
$$

(iii) If characteristic $k$ is inspected after characteristic $j$, the resulting value function is

$$
f_{i-1}(\Theta \backslash\{j\})=p_{k}(\Theta \backslash\{j\}) f_{i-2}(\Theta \backslash\{j\} \backslash\{k\})+c_{k} .
$$

(iv) The probability that both characteristics $j$ and $k$ will be classified fit, given that all characteristics in $\bar{\Theta}$ were classified fit, is

$$
p_{j, k}(\Theta)=\operatorname{Prob}\left\{\left\{X_{j}=1 \cap X_{k}=1 \mid x_{i}=1, \forall i \in \bar{\Theta}\right\} .\right.
$$


This can be written in two ways, providing the identity

$$
\begin{aligned}
p_{j, k}(\Theta) & =p_{j}(\Theta) p_{k}(\Theta \backslash\{j\}) \\
& =p_{k}(\Theta) p_{j}(\Theta \backslash\{k\}) .
\end{aligned}
$$

Combining Eqs. (12) through (15), and noting that Proposition 3.1 implies that

$$
f_{i-2}(\Theta \backslash\{k\} \backslash\{j\})=f_{i-2}(\Theta \backslash\{j\} \backslash\{k\}),
$$

gives the result of the theorem.

\section{Independent Characteristics}

If all the characteristics are independent, then

$$
P_{W}\left(w_{1}, w_{2}, \ldots, w_{N}\right)=\prod_{i} P_{W_{i}}\left(w_{i}\right),
$$

in which case

$$
\begin{aligned}
& b(\Theta)=\operatorname{Prob}\left\{X_{i}=1, \forall i \in \bar{\Theta}\right\}=\prod_{i \in \bar{\Theta}}\left(1-R_{i}\right), \\
& a_{k}(\Theta)=\prod_{r \in \bar{\Theta} \cup\{k\}}\left(1-R_{r}\right),
\end{aligned}
$$

with

$$
R_{i}=P_{i}\left(1-\beta_{i}\right)+\left(1-P_{i}\right) \alpha_{i} .
$$

Then, condition (11) becomes

$$
\begin{aligned}
& c_{k} /\left[\prod_{r \in \bar{\Theta}}\left(1-R_{r}\right)-\prod_{r \in \bar{\Theta} \cup\{k\}}\left(1-R_{r}\right)\right] \\
& \leq c_{j} /\left[\prod_{r \in \bar{\Theta}}\left(1-R_{r}\right)-\prod_{r \in \bar{\Theta} \cup\{j\}}\left(1-R_{r}\right)\right],
\end{aligned}
$$

which reduces to

$$
c_{k} / R_{k} \leq c_{j} / R_{j},
$$

the same result reported in Ref. 2.

\section{Conclusions and Comments}

The problem of sequencing the inspection of characteristics with dependent fitness is addressed by using the results of Theorem 4.1, which reduces 
to the rule given in Ref. 2 for independent characteristics. In particular, expression (11) allows a determination (at any point in the inspection process, when a set $\bar{\Theta}$ has already been inspected) of the optimal next characteristic to inspect. This special characteristic $\left(i^{*}\right)$ is given by

$$
i^{*}=\arg \min c_{i} /\left[1-p_{i}(\Theta)\right]
$$

Moreover, evaluation of $p_{i}(\Theta)$ has computational complexity no worse than $N 2^{N}$. Of course, the dynamic programming equation (5) can also be used, for small $N$, to compute the resulting minimum expected cost. However, as is common with DP in general, an a priori evaluation of the optimal cost function $f_{N}(C)$ cannot be provided for arbitrary input data $P_{W}(\cdot), c_{i}, \alpha_{t}$, $\beta_{i}$, and for large $N$ the associated computation may become excessive.

\section{References}

1. Raouff, A., Jain, J. K., and Sathe, P. T., A Cost Minimization Model for Multicharacteristic Component Inspection, IIE Transactions, Vol. 15, No. 3, pp. 187-194, 1983.

2. Duffuua, S. O., and Raouf, A., An Optimal Sequence in Multicharacteristic Inspection, Journal of Optimization Theory and Applications, Vol. 67, No. 1, pp. 79-86, 1990.

3. Lawler, E. L., and Sivazlian, B. D., Minimization of Time-Varying Costs in Single-Machine Scheduling, Operations Research, Vol. 26, No. 4, pp. 563-569. 1978.

4. Alidaee, B., On Optimal Ordering Policy of a Sequential Model, Journal of Optimization Theory and Applications, Vol. 89, No. 3, pp. 771-776, 1996.

5. Duffuua, S. O., An Optimal Ordering Rule for a Stochastic Sequencing Model, Journal of Optimization Theory and Applications, Vol. 83, No. 1, pp. 199-205, 1994.

6. Dreyfus, S. B., and LAw, A. M., The Art and Theory of Dynamic Programming, Academic Press, New York, New York, 1977. 